\title{
Fabric Evolution and Its Effect on Strain Localization in Sand
}

\author{
Zhiwei Gao and Jidong Zhao
}

\begin{abstract}
Fabric anisotropy affects importantly the overall behaviour of sand including its strength and deformation characteristics. While both experimental and numerical evidence indicates that soil fabric evolves steadily with the applied stress/strain, how evolving fabric influences the initiation and development of shear band in sand remains an intriguing question to be fully addressed. In this paper, we present a numerical study on strain localization in sand, highlighting the special role played by soil fabric and its evolution. In particular, a critical state sand plasticity model accounting for the effect of fabric and its evolution is used in the finite element analysis of plane strain compression tests. It is found that the initiation of shear band is controlled by the initial fabric, while the development of shear band is governed by two competing physical mechanisms, namely, the structural constraint and the evolution of fabric. The evolution of fabric generally makes the sand response more coaxial with the applied load, while the structural constraint induced by the sample ends leads to more inhomogeneous deformation within the sand sample when the initial fabric is non-coaxial with the applied stress. In the case of smooth boundary condition, structural constraint dominates over the fabric evolution and leads to the formation of a single shear band. When the boundary condition is rough, the structural constraint may play a comparable role with fabric evolution, which leads to symmetric cross-shape shear bands. If the fabric is prohibited from evolving in the latter case, a cross-shape shear band pattern is found with the one initiated first by the structural constraint dominant over the second one.
\end{abstract}

\section{Z. Gao $(\bowtie)$}

School of Engineering, University of Glasgow, Rankine Building, Oakfield Avenue, Glasgow G12 8LT, UK

e-mail: Zhiwei.Gao@glasgow.ac.uk

J. Zhao

Department of Civil and Environmental Engineering, Hong Kong University of

Science and Technology, Clearwater Bay, Kowloon, Hong Kong 


\section{Introduction}

Strain localization is frequently observed in sand and is considered an important precursor of the failure of soil and relevant geostructures. Numerous investigations have been carried out on strain localization in sand but relatively less attention has been paid to the correlation of strain localization with the presence of an evolving fabric (e.g., Borja et al. 2013). Fabric anisotropy has been widely regarded to affect the key behaviour of sand including dilatancy, liquefaction and critical state. Based on plain strain compression tests, Tatsuoka et al. (1990) found that the shear band development in sand was dependent on the initial bedding plane orientation, or the fabric, of the sample. Meanwhile, micromechanical studies indicate that fabric evolves steadily with deformation and the evolving fabric exhibits unique characteristics when a sand sample reaches liquefaction, critical state and other states (Guo and Zhao 2013; Zhao and Guo 2013a, b). As an important type of instabilities, strain localization has been commonly observed in sand. The correlation between fabric and fabric evolution is an interesting topic but remains less explored. Inherent fabric anisotropy has recently been considered in a hypoplastic model by Tejchman et al. (2007) in the simulation of shear band development in sand but the interplay between fabric evolution and the development of shear band has not been properly considered. In this study, we employ an anisotropic sand model developed recently by the authors (Gao et al. 2014) and finite element method to investigate strain localization in sand under plane strain compression wherein the special role of fabric and its evolution is highlighted.

\section{Finite Element Analysis of Strain Localization in Plane Stain Compression}

The constitutive model used in this study was developed by Gao et al. (2014). It has been implemented in the finite element package ABAQUS through the user-material interface. The test data presented by Tatsuoka et al. (1990) will be employed to benchmark the model simulations. The model parameters have been calibrated based on the plane strain test results on Toyoura sand. The initial degree of anisotropy $F_{0}$ is set to be 0.45 . The model simulations for single element tests can be found in Gao et al. (2014) and Gao and Zhao (2013).

In the finite element analysis, the sample setup is the same as that in Tatsuoka et al. (1990) (Fig. 1). Uniform 4-noded plane strain elements of $2.5 \mathrm{~mm} \times 2.5 \mathrm{~mm}$ in size are used and constant confining pressure is applied in the horizontal direction of the sample. Vertical displacement $\Delta h$ is applied to the top end of the sample by increment to ensure quasi-static loading. Both smooth (the top and bottom ends are free to move horizontally) and rough (the horizontal displacements of the top and bottom ends are restricted) boundary conditions with and without fabric evolution are considered and the initial void ratio distribution is assumed to be uniform throughout the entire sample. 


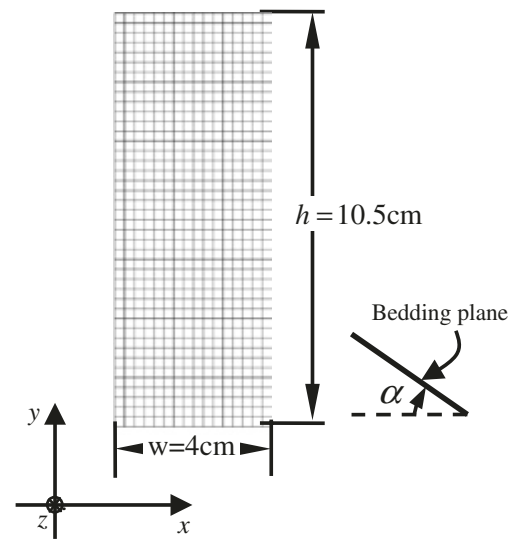

Fig. 1 Sample setup, element size, orientation of bedding plane and the reference coordinate system

(a)

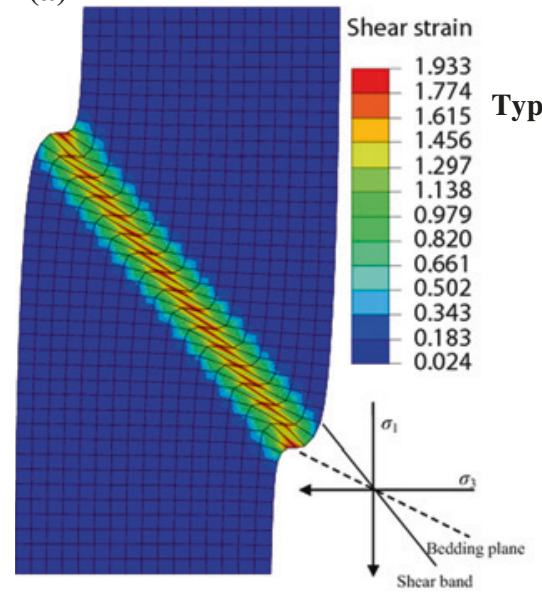

(b)



Fig. 2 Predicted strain localization for the cases with smooth boundary condition and a evolving fabric; b constant fabric at vertical strain $\varepsilon_{h}=\Delta h / h=12 \%$

Figure 2 shows the predicted shear band pattern in smooth boundary condition cases with initial void ratio $e_{0}=0.7$, confining pressure $\sigma_{c}=400 \mathrm{kPa}$ and bedding plane orientation $\alpha=45^{\circ}$. It can be seen that the simulated shear band and bedding plane lie on the same side (left side in the upper part of the sample for the present study) of the major principal stress direction (Fig. 2a) and Tatsuoka et al. (1990) terms similar shear band pattern they observed in laboratory tests as Type-b shear band. Indeed, Type-b shear band is the most commonly observed pattern in laboratory tests with smooth boundary (Tatsuoka et al. 1990). When the fabric is assumed constant (the fabric is prohibited from evolving with deformation), the 

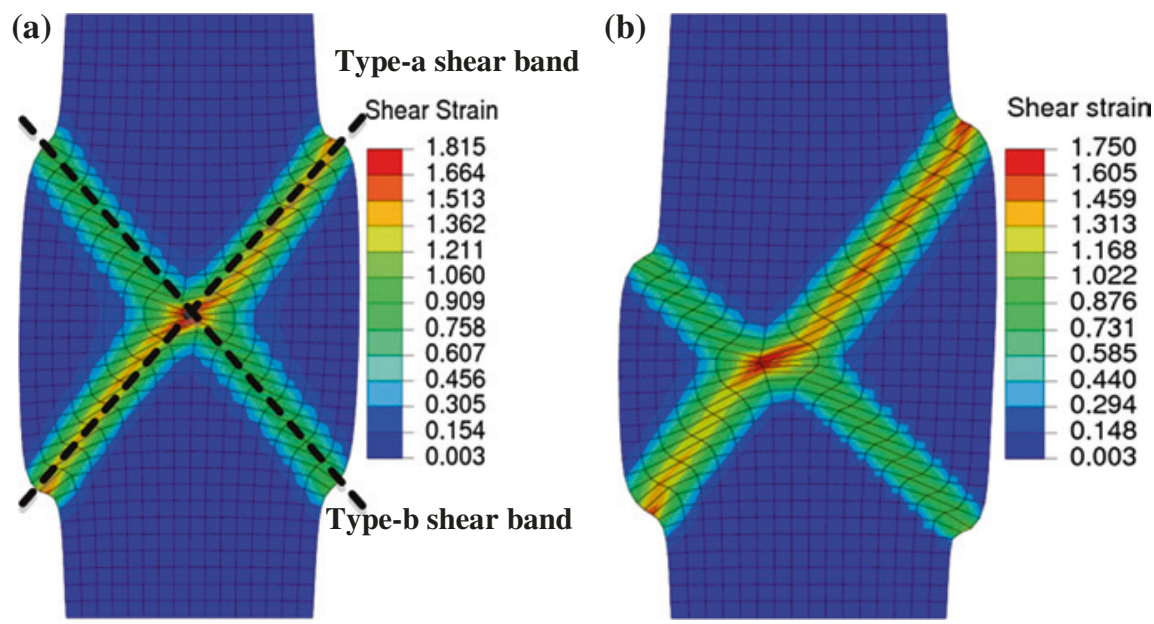

Fig. 3 Predicted strain localization for the cases with rough boundary condition and a evolving fabric; b constant fabric at vertical strain $\varepsilon_{h}=\Delta h / h=15.5 \%$

strain concentration in the shear band (Fig. 2b) will be more intense than the fabric evolution case (Fig. 2a). In addition, it is observed that the predicted vertical strain level corresponding to shear band initiation is independent of initial bedding plane orientation if the fabric is assumed constant, which appears to be inconsistent with the observations in Tatsuoka et al. (1990). This indicates that fabric evolution should be properly accounted for in the strain localization analysis. Figure 3 shows the predicted shear band pattern in rough boundary condition cases $\left(e_{0}=0.7\right.$, $\sigma_{c}=400 \mathrm{kPa}$ and $\alpha=45^{\circ}$ ). Two symmetric and asymmetric shear bands are observed for the cases with and without fabric evolution, respectively. The shear strain level in Type-a shear bands [according to the definition by Tatsuoka et al. (1990)] is higher than that in the Type-b shear bands in both cases.

\section{Mechanisms Governing the Shear Band Patterns}

Our study shows that the development of a shear band is essentially governed by two competing mechanisms - the fabric evolution which reduces the non-coaxial strain increment when the fabric and applied stress are initially non-coaxial and alleviates the strain localization and the structural constraint imposed by the boundary conditions which promotes the shear band development. The structural constraint can be better described by the reaction forces at the top and bottom sample ends as shown in Fig. 4.

In the smooth boundary condition cases, the initial bedding plane constitutes a natural weakened plane along which the sample can develop shear strain concentration and the vertical reaction forces on the two ends drive the upper and lower 
(a)

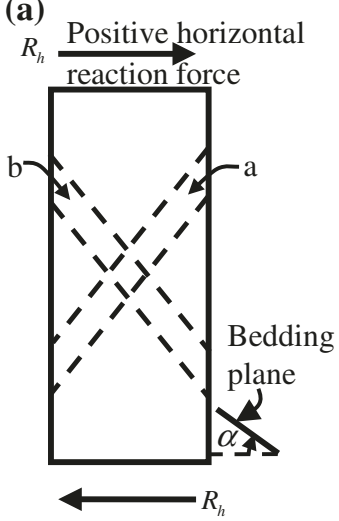

(b)

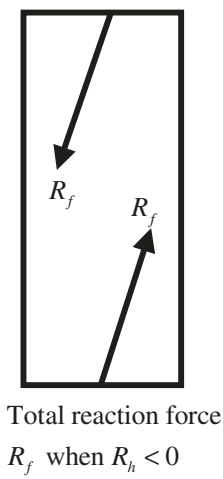

(c)

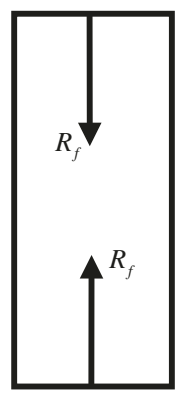

Total reaction force $R_{f}$ when $R_{h}=0$ (d)

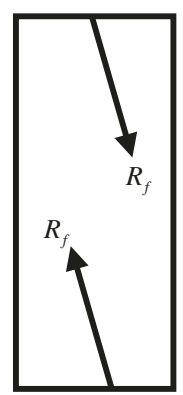

Total reaction force

$R_{f}$ when $R_{h}>0$

Fig. 4 Definition of a the positive direction of the horizontal reaction force $R_{h}$, $\mathbf{c}-\mathbf{d}$ three cases of the total reaction force $R_{f}$ imposed on the sample by the boundary

(a)



(b)

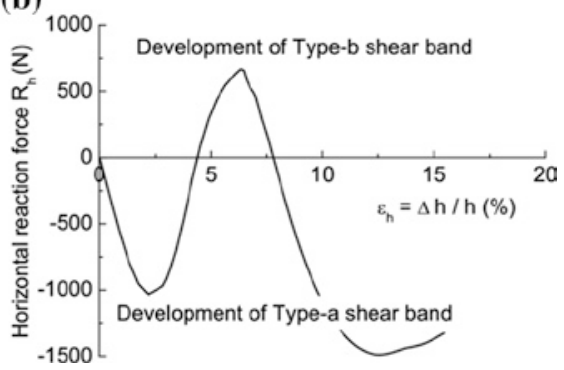

Fig. 5 Evolution of the horizontal reaction force at the top end of the sand sample with $\alpha=45^{\circ}$ and rough boundary condition $\left(e_{0}=0.7, \sigma_{3}=400 \mathrm{kPa}\right)$ : a considering fabric evolution; $\mathbf{b}$ considering constant fabric

parts of the sample to the right and left respectively. This causes the occurrence of a single Type-b shear band. Since the strain localization initiates at relatively low strain level, the effect of fabric evolution is not large enough to prevent the shear band development in this case.

In the rough boundary condition cases, negative $R_{h}$ develops to prevent the horizontal displacement of the top and bottom ends due to the non-coaxial strain increment at the initial loading stage (Fig. 5), which leads to a Type-a shear band initially. As the sample is weakened along direction-a due to stain localization (Fig. 5a), the magnitude of $R_{h}$ decreases and becomes positive subsequently. This causes the initiation of Type-b shear band. Meanwhile, the evolution of fabric can eventually lead to rather symmetric geometry of the two bands as well as sample shape (Fig. 4a). If the fabric is fixed without evolution, the final sample shape and shear bands are asymmetric as $R_{h}$ will become negative again later on (Fig. $5 \mathrm{~b}$ ). 


\section{Conclusions}

The effect of fabric evolution on strain localization in plane strain compression has been studied numerically. It is found that the evolution of fabric generally tends to make the sand response more coaxial with the applied stress and alleviate the strain localization, while the structural constraint induced by the sample ends leads to more inhomogeneous deformation within the sand sample when the initial fabric is non-coaxial with the applied stress. In smooth boundary condition cases, structural constraint dominates over the fabric evolution and leads to the formation of a single shear band. When the boundary condition is rough, the structural constraint may play a comparable role with fabric evolution, which leads to symmetric cross-shape shear bands. If the soil fabric is not allowed to evolve, asymmetric cross-shape shear bands develop.

Acknowledgements The study was financially supported by RGC/GRF 622910 and DAG08/09.EG04.

\section{References}

Borja RI, Song XY, Rechenmacher AL, Abedi S, Wu W (2013) Shear band in sand with spatially varying density. J Mech Phys Solids 61:219-234

Gao ZW, Zhao JD (2013) Strain localization and fabric evolution in sand. Int J Solid Struct 50:3634-3648

Gao ZW, Zhao JD, Li XS, Dafalias YF (2014) A critical state sand plasticity model accounting for fabric evolution. Int J Numer Anal Meth Geomech 38:370-390

Guo N, Zhao JD (2013) The signature of shear induced anisotropy in granular media. Comput Geotech 47:1-15

Tatsuoka F, Nakamura S, Huang CC, Tani K (1990) Strength anisotropy and shear band direction in plane strain tests of sand. Soils Found 30(1):35-54

Tejchman J, Bauer E, Wu W (2007) Effect of fabric anisotropy on shear localization in sand during plane strain compression. Acta Mech 189:23-51

Zhao JD, Guo N (2013a) Unique critical state characteristics in granular media considering fabric anisotropy. Géotechnique 63(8):695-704

Zhao JD, Guo N (2013b) A new definition on critical state of granular media accounting for fabric anisotropy. In: Powders and grains 2013: AIP conference proceedings, vol 1542, pp 229-232. doi: 10.1063/1.4811909 